\title{
Cavitojejunostomy for post-traumatic disconnected pancreatic duct syndrome presenting as persistent external pancreatic fistula: A case report
}

\author{
Kelu Sreedharan Sreesanth ${ }^{1}$, Valiya Kambrath Prathapan ${ }^{2}$, Mathew Joseph ${ }^{3}$, Chandran Nirmala Vyshakh ${ }^{3}$ \\ From ' Assistant Professor, 2Professor, Department of Surgical Gastroenterology, '3 Assistant Professor, Department of General Surgery, Government \\ Medical College, Kozhikode, Kerala, India
}

\begin{abstract}
Post-traumatic disconnected pancreatic duct syndrome (DPDS) can present as persistent external pancreatic fistula following percutaneous drainage (PCD) of pancreatic collection. Management of these cases can be difficult and involves a multidisciplinary approach. Here, we present a case of a 16-year-old boy who presented with persisted pancreatic fistula following initial management including PCD of peripancreatic collection following polytrauma 3 months prior. Magnetic resonance imaging showed a disconnected pancreatic duct. Intraoperatively, there was a disconnected and viable distal pancreas with a defect cavity measuring $2 \times 2 \mathrm{~cm}$ at the neck of the pancreas. The wall of the cavity was anastomosed with a Roux limb of the jejunum (cavitojejunostomy). Post-operative recovery was uneventful and the patient remains asymptomatic at 2 months of follow-up. Cavitojejunostomy is a feasible and safe surgical option in DPDS with a well-formed cavity. This avoids dissection in difficult anatomical planes and preserves pancreatic parenchyma.
\end{abstract}

Key words: Cavitojejunostomy, Disconnected pancreatic duct syndrome, Pancreatic trauma

$\mathrm{D}$ isconnected pancreatic duct syndrome (DPDS) represents a set of clinical conditions that arise due to persistent output from viable distal pancreatic gland segment excluded from the rest of the enteric tract [1]. Common etiological factors for DPDS are necrotizing pancreatitis, pancreatic trauma, chronic pancreatitis, and post-surgical complications [2]. DPDS often leads to persistent pancreatic fistula or collection and diagnosis and management often get complicated as simple percutaneous drainage (PCD) is not sufficient. The management of DPDS usually involves a multidisciplinary approach including endoscopic or surgical internal drainage or resection of the distal pancreas [3]. Although resection of the distal pancreas, pancreaticojejunostomy, and fistula-jejunostomy are some of the available options, surgery is often difficult in these cases due to difficult anatomical planes.

Here, we present a case report of a child who presented with persistent pancreatic fistula following PCD of the pancreatic collection after Type 3 pancreatic injury and was managed surgically with cavitojejunostomy. Cavitojejunostomy as an internal drainage method has not been well described in the literature. Here, we highlight the usefulness of cavitojejunostomy in DPDS without drainable collection and reviewed on various treatment options of DPDS management.

\section{Access this article online}

Received - 14 October 2021

Initial Review - 29 October 2021

Accepted - 16 November 2021

DOI: $10.32677 /$ ijcr.v7i11.3131

\section{CASE REPORT}

A 16-year-old male child had an alleged history of a road traffic accident 3 months back. He was the pillion driver when the twowheeler was hit by a four-wheeler. The child sustained polytrauma to the head, chest, and abdomen. There was no history of loss of consciousness and the child was immediately shifted to a nearby hospital where he underwent initial evaluation and treatment.

On evaluation with computerized tomography, he was found to have Grade 2 liver injury and Grade 3 pancreatic injury along with multiple vertebral bone fractures and hematoma inferior to the right kidney. The patient was initially managed conservatively but emergency laparotomy was done on post-admission day 1 in view of unstable vitals and fall in hemoglobin ( 9 to $6.5 \mathrm{~g} \%$ ). Intraoperative findings were non-expanding retroperitoneal hematoma, non-bleeding liver laceration in segment 4 , and contusion at the head and neck of the pancreas without any capsular tear or evidence of pancreatic fluid lead. Peritoneal wash was given and the abdomen was closed. The patient was shifted to an intensive care unit and his clinical conditions improved.

Magnetic resonance imaging done 6 days after injury showed complete pancreatic parenchymal discontinuity at the neck of pancreas suggestive of Grade III laceration with an intra- and peri-pancreatic collection of size $6.6 \times 6.2 \times 9.1 \mathrm{~cm}$ (Fig. 1). The

\footnotetext{
Correspondence to: Kelu Sreedharan Sreesanth, Department of Surgical Gastroenterology, Government Medical College, Kozhikode, Kerala, India. E-mail: sreesanth48@gmail.com

(C) 2021 Creative Commons Attribution-NonCommercial 4.0 International License (CC BY-NC-ND 4.0).
} 


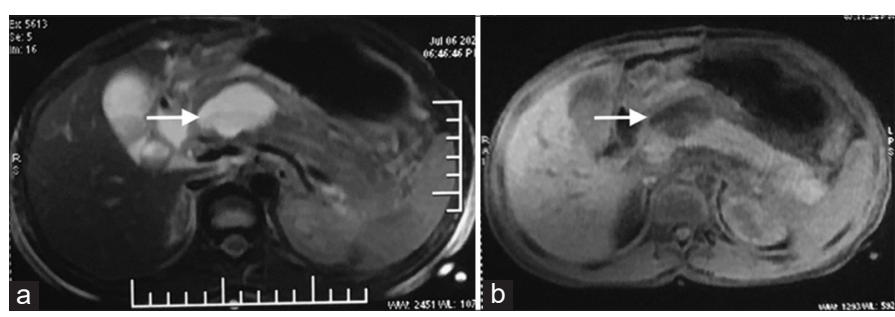

Figure 1: (a) T2-weighted magnetic resonance imaging image showing fluid-filled cavity at neck of pancreas; (b) T1-weighted image showing completed gland discontinuity at neck of pancreas

patient developed abdominal distention and raise in bilirubin level on post-operative day 10 and an ultrasound examination of the abdomen showed a $9.1 \times 8.6 \mathrm{~cm}$ collection at the region of the head of the pancreas. In view of clinical symptoms and increase in the size of the collection, a PCD was placed in the collection which drained around $300 \mathrm{ml}$ of serous fluid with a high amylase level. His symptoms improved except for the persistent daily output of around $300 \mathrm{ml}$ through the drain. In view of the persistent drain output, he was referred to our center for further management.

Here, he underwent endoscopic retrograde cholangiopancreatography (ERCP) which showed pancreatic duct cutoff at the neck with spillage of contrast. Transpapillary stenting was tried but was unsuccessful. A follow-up ultrasound examination showed a $2 \times 2 \mathrm{~cm}$ collection at the neck of the pancreas. In view of persistent fistula and failed ERCP, he was planned for surgical management 2 months after the first surgery.

The abdomen was opened along the previous incision scar. Intraperitoneal adhesions due to the previous laparotomy were noted. Careful adhesiolysis was done and the percutaneous drain track was followed to reach a cyst at the neck of the pancreas. On further dissection, there was a $2 \times 2 \mathrm{~cm}$ well-formed cavity at the neck of the pancreas showing continuous filling of pancreatic juice (Fig. 2). The rest of the pancreas was normal. Forty centimeters Roux limb was taken retrocolically and anastomosed to the periphery of the cavity using interrupted 4-0 PDS sutures (Fig. 3) and a perianastomotic drain was placed and the abdomen was closed. The post-operative period was uneventful and the drain was removed on post-operative day 3 as the output was minimal and serous. The patient was discharged on post-operative day 5 in a stable condition. At 2 months of follow-up, the patient remains asymptomatic.

\section{DISCUSSION}

The term DPDS was introduced by Kozarek et al. [4] for clinical conditions resulting from a persistent leak from the viable distal pancreas which is disconnected from the rest of the gastrointestinal tract. It occurs when the main pancreatic duct is necrosed and upstream pancreatic gland tissue remains viable to produce pancreatic juice. Common predisposing factors are acute necrotizing pancreatitis, pancreatic trauma, chronic pancreatitis, and post-surgical complications [2].

DPDS or Type 3 ductal injury is further subclassified into Type $3 \mathrm{~A}$ or $3 \mathrm{~B}$ depending on whether the distal duct is occluded

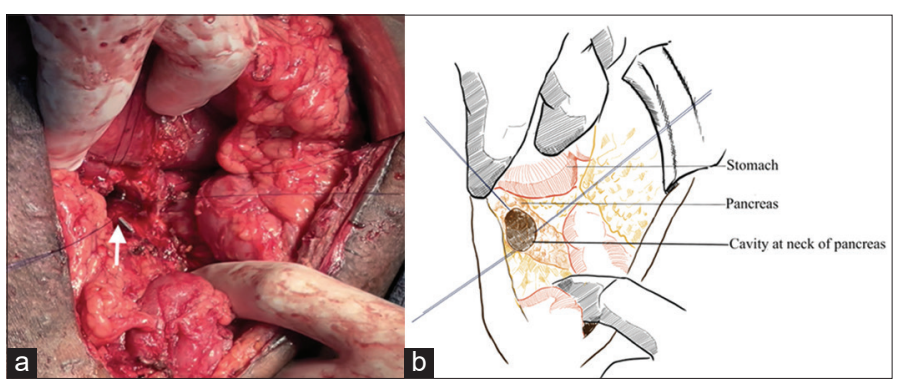

Figure 2: (a) Intraoperative picture of cavity at pancreatic neck; (b) schematic illustration of cavity at the pancreatic neck
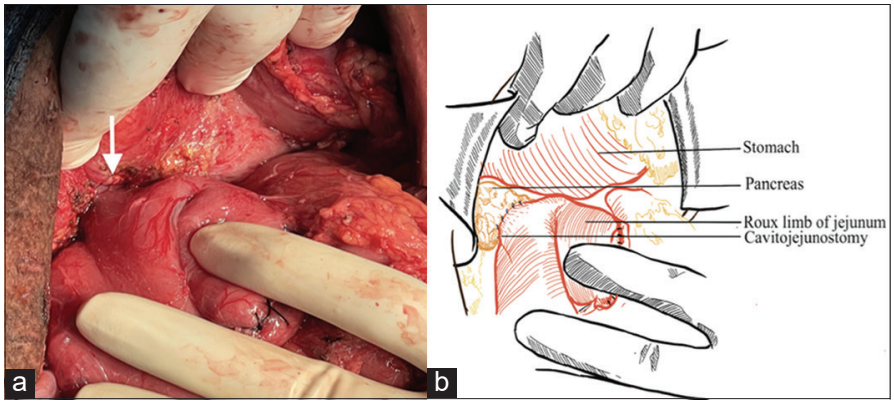

Figure 3: (a) Intraoperative image of completed cavitojejunostomy; (b) schematic illustration of completed cavitojejunostomy

or draining freely, respectively [5]. The index patient had type 3B ductal injury following blunt abdominal trauma. Incidence of pancreatic injury following blunt abdominal trauma is in the range of $0.4-12 \%$ with nearly $1.3 \%$ being high-grade injury [3]. Distal pancreatectomy (DP) is the standard management option for type 3 injury but there has been increasing interest in nonoperative management lately, especially in children $[3,6]$.

DPDS can present with pancreatic ascites, pseudocyst, persistent pancreatic fistula, or pancreatic-pleural fistula. The diagnosis is often made by contrast-enhanced computed tomography, magnetic resonance cholangiopancreatography with or without secretin enhancement, or ERCP. Two centimeters or greater necrosis of the pancreatic duct with a viable upstream pancreatic gland in imaging study and pancreatic duct cutoff in ERCP suggest DPDS [7].

DPDS management can be extremely difficult and require a multidisciplinary approach including radiological, endoscopic, or surgical interventions for successful outcomes [8,9]. In some patients with small asymptomatic collection, conservative management may be tried. Rarely, the upstream pancreas may undergo atrophy and the collection may resolve $[5,10]$. Isolated PCD is likely to result in persistent external pancreatic fistula and hence should be avoided [11]. Endoscopic options include transpapillary or transmural route drainage, or dual-modality drainage (transmural drainage along with PCD) [10]. Although the initial success rate is nearly $80 \%$, long-term failure rates are more with endoscopic treatment options [12]. In cases with persistent external fistula, transmural drainage may not be feasible as the drainable collection may not be present [10]. Minimally invasive endotherapy is often the preferred initial therapy, but surgical intervention has a definitive role in the management of DPDS. 
Surgical options include resection of the distal pancreas with or without splenic preservation, or internal enteric drainage of the distal pancreas, pseudocyst, or the fistulous tract. Amount of disconnected gland, peripancreatic inflammation, the exocrine and endocrine function of the pancreas, size and the location of pancreatic fluid collection, and the presence of sinistral portal hypertension determine the choice of surgical procedures. In an appropriate clinical setting, success rates up to $74-82 \%$ can be achieved with distal pancreatic resection or internal drainage [13]. A study by Dokmak et al. [14] in 36 patients of DPDS, managed with pancreatoenteric reconnection, showed a clinical success rate of $91 \%$ and a low rate of de novo exocrine and endocrine insufficiency. They mobilized $3 \mathrm{~cm}$ of the distal pancreas and were anastomosed to the stomach after freshening the margin. Clinically, the significant post-operative pancreatic fistula rate was $36 \%$ and the mortality rate was $3 \%$. The enteric anastomosis can also be done to the fistulous tract in cases where mobilization of the distal pancreas is difficult. One study comparing fistulojejunostomy and DP found that DP had increased blood loss, transfusion requirement, and deterioration of endocrine function, whereas, fistulojejunostomy resulted in more persistent fistula requiring reoperation [15]. Surgery in DPDS is a difficult game due to inflammation, venous hypertension, fibrosis, etc. [13]. Cavitojejunostomy is a relatively safe option in a setting of a well-formed cavity and inflamed and fibrotic anatomical planes, which may prevent safe dissection of the pancreas. In our case, we could anastomose roux limb of jejunum to the cavity at the neck of the pancreas with good outcomes.

\section{CONCLUSION}

Grade 3 pancreatic injury may present as DPDS following initial conservative management. DPDS presenting as persistent pancreatic fistula is often difficult to manage as transmural drainage is not possible always and often requires a multimodality approach. Although minimally invasive endoscopic therapy is the preferred initial therapy in DPDS, surgical options might be required in cases where endotherapy fails. Cavitojejunostomy is a relatively safe option with good outcomes in patients with a wellformed cavity at the site of ductal injury. Unlike other surgical options such as pancreatojejunostomy or DP, this avoids the need for a dissection through difficult anatomical planes and preserves pancreatic parenchyma.

\section{REFERENCES}

1. Nadkarni NA, Kotwal V, Sarr MG, Swaroop Vege S. Disconnected pancreatic duct syndrome: Endoscopic stent or surgeon's knife? Pancreas 2015;44:16-22.

2. Devière J, Bueso H, Baize M, Azar C, Love J, Moreno E, et al. Complete disruption of the main pancreatic duct: Endoscopic management. Gastrointest Endosc 1995;42:445-51.

3. Agarwal H, Gupta A, Kumar S. An overview of pancreatic trauma. J Pancreatol 2020;3:139-46.

4. Kozarek RA. Endoscopic therapy of complete and partial pancreatic duct disruptions. Gastrointest Endosc Clin N Am 1998;8:39-53.

5. Nealon WH, Bhutani M, Riall TS, Raju G, Ozkan O, Neilan R. A unifying concept: Pancreatic ductal anatomy both predicts and determines the major complications resulting from pancreatitis. J Am Coll Surg 2009;208:790-9; discussion 799-801.

6. Søreide K, Weiser TG, Parks RW. Clinical update on management of pancreatic trauma. HPB (Oxford) 2018;20:1099-108.

7. Sandrasegaran K, Tann M, Jennings SG, Maglinte DD, Peter SD, Sherman S, et al. Disconnection of the pancreatic duct: An important but overlooked complication of severe acute pancreatitis. Radiographics 2007;27:1389-400.

8. Fischer TD, Gutman DS, Hughes SJ, Trevino JG, Behrns KE. Disconnected pancreatic duct syndrome: Disease classification and management strategies. J Am Coll Surg 2014;219:704-12.

9. Machado NO. Disconnected duct syndrome: A bridge to nowhere. Pancreat Disord Ther 2015;5:153.

10. Larsen M, Kozarek RA. Management of disconnected pancreatic duct syndrome. Curr Treat Options Gastroenterol 2016;14:348-59.

11. Maatman TK, Mahajan S, Roch AM, Ceppa EP, House MG, Nakeeb A, et al. Disconnected pancreatic duct syndrome predicts failure of percutaneous therapy in necrotizing pancreatitis. Pancreatology 2020;20:362-8.

12. Irani S, Gluck M, Ross A, Gan SI, Crane R, Brandabur JJ, et al. Resolving external pancreatic fistulas in patients with disconnected pancreatic duct syndrome: Using rendezvous techniques to avoid surgery (with video). Gastrointest Endosc 2012;76:586-93.e1-3.

13. Murage KP, Ball CG, Zyromski NJ, Nakeeb A, Ocampo C, Sandrasegaran K, et al. Clinical framework to guide operative decision making in disconnected left pancreatic remnant (DLPR) following acute or chronic pancreatitis. Surgery 2010;148:847-56; discussion 856-7.

14. Dokmak S, Tetart A, Aussilhou B, Choquet A, Rebours V, Vullierme MP, et al. French reconnection: A conservative pancreato-enteric reconnection for disconnected pancreatic duct syndrome. Pancreatology 2021;21:282-90.

15. Dhar VK, Sutton JM, Xia BT, Levinsky NC, Wilson GC, Smith M, et al. Fistulojejunostomy versus distal pancreatectomy for the management of the disconnected pancreas remnant following necrotizing pancreatitis. J Gastrointest Surg 2017;21:1121-7.

Funding: None; Conflicts of Interest: None Stated.

How to cite this article: Sreesanth KS, Prathapan VK, Joseph M, Vyshakh CN. Cavitojejunostomy for post-traumatic disconnected pancreatic duct syndrome presenting as persistent external pancreatic fistula: A case report. Indian J Case Reports. 2021;7(11):497-499. 